\title{
Judgements about fellow professionals and the management of patients receiving palliative care in primary care: a qualitative study
}

\author{
Catherine Walshe, Chris Todd, Ann-Louise Caress and Carolyn Chew-Graham
}

\begin{abstract}
Background

Policies emphasise the importance of collaborative working in community palliative care. Collaborations are generally formed through formal and informal referral processes, but little is known about what influences professionals' decisions to refer to such services.
\end{abstract}

Aim

To explore the influences on referrals within general and specialist community palliative care services.

Design of study

Qualitative, multiple-case study.

Setting

Three primary care trusts in the north-west of England.

Method

Multiple data collection methods were employed including documentary analysis, observation of referral team meetings and interviews. This paper primarily reports data from interviews with 47 health professionals, including GPs, district nurses, and specialist palliative care professionals.

\section{Results}

Judgements - positive and negative - about aspects of fellow professionals' performances appeared to influence referral decisions and ongoing collaboration and care. Attributes upon which these judgements were based included professional responsiveness and communication, respect, working and workload management practices, perceived expertise, and notions of elite practice. The effects of such judgements on referral and healthcare practices were altered by professional 'game playing' to achieve professionals' desired outcomes.

\section{Conclusion}

Palliative care policies and protocols need to take account of these complex and subtle influences on referrals and collaboration. In particular, teamwork and partnership are encouraged within palliative care work, but critical judgements indicate that such partnerships may be difficult or fragile. It is likely that such judgemental attitudes and practices affect many aspects of primary care, not just palliative care.

Keywords

case study; interprofessional care; palliative care primary health care; qualitative research.

\section{INTRODUCTION}

A partnership or team approach to palliative care provision is routinely accepted as good practice; it is an inherent feature of the World Health Organisation's definition of palliative care ${ }^{1}$ and emphasised in current policy and guidance..$^{2,3}$ It is assumed that interdisciplinary collaboration will bring about more efficient and effective work and, consequently, that patients will receive better care. ${ }^{4}$ Studies of those with complex or chronic health problems ${ }^{5,6}$ suggest improved care through collaboration, but there are few studies examining the impact of collaborative or team-working practices on the process or outcomes of palliative care. ${ }^{3}$

In the UK, providers of community palliative care services, such as GPs, district nurses, allied health professionals, and specialist palliative care professionals, rarely work with each other in formally constituted teams. Instead, a network of services is formed and re-formed constantly around patients,

C Walshe, BNurs, RGN, DNcert, MSc, PhD, RCUK research fellow; C Todd, BA, MA, PhD, CPsychol, AFBPsySoc, professor of primary care and community health; A-L Caress, BNurs, $R G N, H V c e r t, D N c e r t, P h D$, senior lecturer in nursing, School of Nursing, Midwifery and Social Work, University of Manchester. C Chew-Graham, MD, FRCGP, senior lecturer in primary care, School of Community-Based Medicine, University of Manchester; and National School for Primary Care Research, National Institute of Health Research, UK, Manchester.

Address for correspondence

Dr Catherine Walshe, The University of Manchester, The School of Nursing, Midwifery and Social Work, University Place, Oxford Road, Manchester, M13 9PL. E-mail: catherine.walshe@manchester.ac.uk.

Submitted: 13 June 2007; Editor's response: 16 August 2007; final acceptance: 19 November 2007.

(OBritish Journal of General Practice.

This article was originally online first. Cite this article as: Br J Gen Pract 2008; 58: 264-272. Advance online publication. DOI: 10.3399/bjgp08X279652 
based on formal or informal referrals or discussions about patient care plans.

It is not clear whether such formal and informal referrals result either in effective collaborative working or appropriate and equitable service utilisation. It appears that patients receiving palliative care do not access community-based services equitably. Older people from some black and minority ethnic communities and those people without a cancer diagnosis access services less frequently. ${ }^{7.8}$ Most research in this area has explored the disparities themselves, rather than examining why such apparent inequity in access occurs. It has been hypothesised that patients' attitudes to, or awareness of, services may affect access. ${ }^{9,10}$ Characteristics of the healthcare system, including how people collaborate, could also affect whether and how patients receive services. ${ }^{11}$

Professionals' knowledge and skills can affect referral patterns. They may refer to compensate for their own skill deficits ${ }^{12}$ or lack knowledge about those services that are offered. ${ }^{13}$ Prognostication difficulties in palliative care mean that professionals may deliberately delay discussing palliative care services until a late stage in the patient's illness. ${ }^{14}$ Professionals appear positive about the concept of specialist palliative care services ${ }^{15}$ but may not be comfortable about the reality of referring patients to such services. ${ }^{16}$ They may also believe that they are providing good-quality care without the need to refer to, and therefore collaborate with, palliative care services. Those working in nursing homes as district nurses and GPs all express some reluctance to refer patients to specialist palliative care services because they feel they are able to provide good care themselves. ${ }^{17-19}$

There is no previous research looking in depth at factors influencing professionals' decisions to make formal or informal referrals within community palliative care services. This article reports the findings from a qualitative study that explored the influences on referrals within general and specialist community palliative care services from multiple stakeholder perspectives.

\section{METHOD}

\section{Research strategy and setting}

A multiple-case-study research strategy was adopted to facilitate the exploration of what was anticipated to be a complex, context-dependent process..$^{20,21}$ The case was defined as 'those services providing community general and specialist palliative care to patients registered or residing within a specified primary care trust'. Three primary care trusts (PCTs) in the north-west of England (A, B, and C) were selected. These three trusts permitted

\section{How this fits in}

Partnership, collaboration, and team working have been strongly encouraged in

both primary and palliative care. Team structures and team processes are

known to affect collaborative working practices. This qualitative study shows

that professionals' critical judgements about others with whom they work and

refer also appear to affect whether or when referrals are made, as well as

ongoing collaborative care. Health professionals should, therefore, pay attention

to team relationships to promote effective referral practices and continued

collaborative working

variability with respect to PCT size and geography, demography, and provision of palliative care - all areas that may be hypothesised to affect access. ${ }^{22}$ Brief contextual details of the trusts selected are given in Table 1.

A range of data sources was chosen: documentary evidence, interviews with health professionals and patients, and observation of team meetings discussing referrals. This article primarily reports data from the interviews with the health professionals, as it was in the interview situations that professionals revealed the impact of their judgements about fellow professionals on their referral practices.

\section{Participants}

Health professionals were invited to participate either because they were identified as knowledgeable informants by other responders or by the researcher during early case-study visits, or because they were professionals who made or received palliative care referrals. Key informants (that is, those in particular managerial or commissioning posts) and most specialist health professionals were purposively selected: other participants such as district nurses and GPs were identified from staff lists.

Health professionals within case-study sites were invited to participate by letter, with non-responders followed up by letter and telephone. Recruitment ceased when either no new themes emerged from data analysis within that trust or all of its relevant informants were recruited.

\section{Data collection}

Interviews lasted between 50 and 90 minutes. The interviews were open-ended and conversational in style, but with a topic guide derived from the research questions, reviewed literature, and which iteratively developed and changed throughout the study. Participants gave written consent to participate and to their interview being audiotaped. Detailed contemporaneous field notes were made and tape-recordings of the interviews fully transcribed. 
Table 1. Features of the three case-study sites.

\begin{tabular}{|c|c|c|c|c|c|c|}
\hline Trust A & $\begin{array}{c}\text { Small PCT } \\
\text { serving an } \\
\text { industrial town }\end{array}$ & $\begin{array}{l}\text { Mainly white } \\
\text { economically } \\
\text { deprived } \\
\text { population }\end{array}$ & $\begin{array}{l}\text { Trust has a } \\
\text { small, developing } \\
\text { inpatient hospice. } \\
\text { Primary care } \\
\text { provision is } \\
\text { historically important }\end{array}$ & $\begin{array}{l}\text { Early adopters } \\
\text { of GSF }\end{array}$ & $\begin{array}{l}\text { Several GPs } \\
\text { with special } \\
\text { interest in } \\
\text { palliative care. } \\
\text { Mostly large } \\
\text { GP practices }\end{array}$ & $\begin{array}{c}\text { All district } \\
\text { nurses are } \\
\text { practice } \\
\text { attached }\end{array}$ \\
\hline Trust B & $\begin{array}{c}\text { Large PCT } \\
\text { serving an } \\
\text { industrial town }\end{array}$ & $\begin{array}{l}10 \% \text { minority } \\
\text { ethnic } \\
\text { population, with } \\
\text { mixed areas of } \\
\text { deprivation and } \\
\text { affluence }\end{array}$ & $\begin{array}{l}\text { Has a large hospice } \\
\text { with comprehensive } \\
\text { special palliative care } \\
\text { provision acting as a } \\
\text { focus of the areas } \\
\text { palliative care }\end{array}$ & Piloting GSF & $\begin{array}{l}\text { No GPs with } \\
\text { special interest in } \\
\text { palliative care. } \\
\text { Mix of large } \\
\text { and small } \\
\text { GP practices }\end{array}$ & $\begin{array}{c}\text { All district } \\
\text { nurses are } \\
\text { practice } \\
\text { attached }\end{array}$ \\
\hline Trust C & $\begin{array}{c}\text { Mid-size PCT } \\
\text { serving an } \\
\text { urban area }\end{array}$ & $\begin{array}{c}30 \% \text { minority } \\
\text { ethnic } \\
\text { population } \\
\text { with all areas } \\
\text { economically deprivec }\end{array}$ & $\begin{array}{c}\text { No specialist } \\
\text { palliative care provision } \\
\text { within the PCT except for } \\
\text { community Macmillan nursing } \\
\text { d team. Referrals made to } \\
\text { out of area hospice }\end{array}$ & No GSF use & $\begin{array}{l}\text { No GPs with } \\
\text { special interest in } \\
\text { palliative care } \\
\text { Many single- } \\
\text { handed GPs }\end{array}$ & $\begin{array}{c}\text { All district } \\
\text { nurses are } \\
\text { practice } \\
\text { attached }\end{array}$ \\
\hline
\end{tabular}

GSF = Gold Standards Framework. PCT = primary care trust.

Data were collected over 20 months between 2003-2005. Trusts were studied sequentially, with data collection commencing first in site $\mathrm{C}$, then site $B$, then site $A$.

\section{Data analysis}

Data collection and analysis were conducted iteratively, with initial analytical insights informing further data collection. Analysis within trusts preceded cross-case comparisons. Techniques from framework analysis facilitated within- and acrosscase analysis, particularly cross-case patternmatching. ${ }^{23}$ An initial thematic framework was developed and systematically applied to the data using NVivo ${ }^{\circledast}$ (version 2.0). Changes were iteratively made to reflect the data collected.

Rigour was promoted within the study by using multiple sources of evidence; establishing a chain of evidence using NVivo to track data; peer debriefing

Table 2. Breakdown of research participants. ${ }^{a}$

\begin{tabular}{lcccc} 
& \multicolumn{3}{c}{ Case-study site } & Total \\
\cline { 2 - 4 } Responder group & A & B & C & 14 \\
\hline District nursing staff & $5(12)$ & $4(8)$ & $5(12)$ & 14 \\
\hline GPs & $3(24)$ & $4(34)$ & $6(27)$ & 13 \\
\hline Allied health professionals & $0(0)$ & $1(1)$ & $0(0)$ & 1 \\
\hline Key informants & $1(2)$ & $1(2)$ & $2(4)$ & 4 \\
\hline Specialist palliative care nurses & $4(5)$ & $3(3)$ & $3(3)$ & 10 \\
\hline Specialist palliative care doctors & $0(1)$ & $1(1)$ & $1(1)$ & 2 \\
\hline Specialist allied health professionals & $0(0)$ & $2(2)$ & $1(1)$ & 3 \\
\hline Total number of interviews & 13 & 16 & 18 & 47 \\
\hline
\end{tabular}

${ }^{a}$ Figures in brackets indicate the number of professionals approached by letter to participate in the study. with members of a research advisory group (including fellow researchers and palliative care professionals); independent reading of transcripts and identification of key themes; critical comment on interpretations; use of ample data extracts to support themes; and systematically relating concepts through use of theoretical propositions, supported in more than one case-study site. ${ }^{21}$

\section{RESULTS}

Forty-seven interviews with health professionals were conducted across the three study sites (Table 2). Data extracts are identified by professional affiliation; case-study identifiers have been removed to protect anonymity.

Judgements, both positive and negative, about aspects of a fellow professional's performance featured heavily in the data. A distinct notion of regarding individuals as 'good' or 'bad' professionals emerged, based on certain attributes. These included:

- responsiveness and communication;

- respect shown towards others;

- working and workload management practices;

- expertise; and

- notions of elite practice.

The impact of any negative judgements was ameliorated by professional 'game playing'. These are discussed in turn.

\section{Responsiveness and communication}

Other health professionals singled out GPs in their comments regarding responsiveness and 
communication. Nurses portrayed GPs as reluctant to respond to patient need, nurse requests, or new initiatives:

'Its not an easy practice to work with. It's very hard to get the GP to go out and do a visit anyway with palliative patients. And I don't know whether this person doesn't feel it's necessary, sometimes, to refer to us where these people who are more compassionate about the whole situation would refer.' (District nurse [DN] 14)

'Well some [GPs] don't do the GSF [Gold Standards Framework], and won't do the GSF, some won't provide anticipatory medication for patients who are in the terminal phases, some won't go out and visit patients.' (DN4)

Other research reveals that some of these difficulties may concern professional boundaries, with GPs reluctant to accept the expertise or direction of others outside their own profession. ${ }^{24}$ Additionally, nurses may need to use medical language to communicate effectively with timepressured GPs. ${ }^{25}$

However, some professionals commented positively about the responsiveness of GPs:

'I don't have any hesitation in asking them [GPs] to go out, they are normally pretty good, they do respond quite quickly.' (DN1)

Others have found that GPs' interest and involvement in palliative care varies,,$^{26}$ and it may be that it is this variation between 'good' and 'bad' responsiveness that is frustrating. Many comments about responsiveness related to communication issues; if there was an easy way to communicate with GPs, this had a positive effect on both responsiveness and an appraisal of that GP:

'Some of the GPs, the GPs here, it's much, much easier, because if they've got a patient in, we'll wait for the next patient and then bob in. More difficult at the other surgery, and they won't, they don't like to speak to you, you have to wait 'til after surgery.' (DN7)

Concepts of 'good' and 'bad' doctors did not necessarily relate to their technical expertise or medical knowledge, but to their willingness to acquiesce and respond to requests. This may be because doctors have the power to disrupt or legitimate nurses' management of patients. ${ }^{27}$ Others have criticised GPs as unwilling to listen, to discuss issues, or to trust the judgement of the nurse. ${ }^{28}$

\section{Respect towards others}

Professionals recognised that their relationships with, and their perceptions of, each other influenced how they worked together and made referrals. They appreciated that efforts were needed to build relationships that facilitated their own working practices and the social cohesion of the 'team':

'The key things are the good relationships between all three people [district nurse, GP and Macmillan nurse] and knowing what people do, and what people do well, and having that trust and understanding of each other's roles really, and their competencies.' (Key informant [KI] 4)

'Having professional respect, and sometimes they might do something, and you think, "Oh God, I can't believe they've been in there and said that to a patient", but that's what that service is about, and you have to kind of work best together.' (DN6)

There was appreciation that relationships between professionals developed over time and endured beyond the care of individual patients. Such relationships appeared to supersede the patient's immediate needs. There was an emphasis on social cohesion of the team above the task cohesion of caring for that individual patient:

'The relationships with GPs is very much "feel your way and find your feet," that way you see just how far you can go and how far you can't go. Because, I think the problem is, once you have blown it with a GP, you've blown it, and that is it, it's not easy to get back in with them.' (Specialist nurse [SN] 6)

Professionals could be judged on the basis of current and past interactions. They were mindful that the successful referral and care of current patients could depend on their negotiation of a continued successful relationship with others. This could mean that the care of current patients was compromised in order to maintain and enhance a professional relationship that would facilitate the referral and care of patients in the future. Even autonomous professionals are dependent on each other and the risks of 'rocking the boat' and unsettling a relationship are often perceived as too high. ${ }^{29}$

The maintenance of this careful relationship with doctors described by nurses does not appear reciprocated in the data from doctors. Although doctors often appreciated the working relationships they had with nurses, and valued their skills and input, these responders did not describe having to 
negotiate care and relationships in the way that nurses did. This may be because of the difference in power the individual professionals had, and the de facto leadership role that doctors tended to assume:

'I think in good practices the GP obviously plays quite a good leadership role in actually helping to kind of bring people together and discuss and facilitate discussion, and tease out management strategies and things. But I think the district nurse is far more knowledgeable than the average GP.' (GP9)

This assumption of a leadership role by medical staff was clearly seen in the team meetings observed, with each either formally or informally chaired by a doctor. The doctors appeared to set the agenda, and lead and steer the meetings, with the nurses having a quiet, subservient role:

'The meeting was dominated by the GPs who took the lead in deciding which patients to discuss, based on a practice-generated cancer register. The district nurse appeared to know most of the patients discussed, but only participated to volunteer information when asked, not of their own volition.' (Field notes from observation of practice palliative care meeting)

It may be that the behaviour of nurses in medically dominated meetings within the practice, is very different to their individual encounters with doctors - it certainly did not reflect their criticisms of doctors they voiced in the interview situation. It is known that nurses are reluctant to criticise the care of others in open meetings. ${ }^{30}$ Doctors, by virtue of their professional culture, may expect to make decisions and be in charge. ${ }^{31}$ The judgemental attitudes that affected whether and when referrals were made and professionals worked together, appeared to be held privately or within disciplines, rather than being openly acknowledged. These were seen as a barrier to the way people worked together.

Working and workload management practices District nurses were most likely to attract judgemental comments about working practices. A concern expressed was whether they were involved in patient care early, with variations and changes in practice noted:

'Before, district nurses picked them up from the beginning, and went through the whole process, so they were just the right support visit, but apparently now they don't like the support visits.' (SN2)
Staffing difficulties triggered some of these challenges:

'So we get to know about patients, um, at diagnosis really, and we haven't got the staff at the moment to be able to follow them all up.' (DN4)

Some difficulties in working practices were triggered by a perceived reluctance of others to make timely referrals:

'Well, if you can't get through to the GP that you need, to be involved well in advance and not just when they need the nursing care, then you are never going to be able to do that good palliative care because you're always going to be crisis managing. So you have to be able to get through to the GP when you need these patients referred to you.' (KI4)

There appeared to be a culture of mutual blame. Some district nurses were negatively judged because they chose not to visit early; some, however, seemed to shift the blame to others because of staffing difficulties or the timeliness of referrals to their services.

\section{Expertise}

The expertise and interest of some district nurses could trigger negative comments:

'You hear cues, you pick up on cues, and you, you take them forward, and I'm not always convinced that district nurses do that. That a patient may say something, they can either block it or pick up on it, and it depends how many more visits they've got in the diary that day.' (SN4)

Again, however, the comment is not just about the absolute expertise of the nurse to provide care, but is tempered by the workload context. It was how district nurses used their expertise and time, which was adversely commented upon.

Although district nurses frequently made negative comments about GPs, GPs more often made positive comments about their district nursing colleagues, frequently referring to the trust they had in them to manage palliative care:

'They will very often manage a patient almost completely; they will deal with a death at home very well I feel.' (GP2)

'They see patients more often, I think they're a bit more proactive than I am, so they tend to see, 
they tend to pick up problems that I've not identified.' (GP5)

This confidence appeared to highlight the nurses' functional authority, gained because of their knowledge about, and relationship with, patients. Such assessment and relationship-building work, if undertaken by district nurses, also saves GPs from having to see and assess patients. ${ }^{32}$

\section{Elite practice}

The issue of elite practice was particularly evident in comments about the specialist-nursing service in one case-study site:

'They'd rather go off and work in a team where they can be "proper specialist palliative care people", and restrict the numbers of patients they see, and put in special referral protocols, so they, they don't necessarily see everybody, and they become elitist about the care they offer, and all of that kind of thing, that specialist palliative care people do ... ultimately when that kind of relationship with the Macmillan nurses is going on, I mean, ultimately, you kind of forget to refer.' (GP3)

In contrast, no negative comments were made about the specialist nurses in a different case-study site, who appeared to work hard at developing relationships founded on 'referral etiquettes' with generalist professionals.

The differences between the sites highlighted the contextual differences between what appeared, to an outside observer, to be virtually identically provided services. It was the approaches of the different individuals within those services, and the impact this had on the culture within their teams, that appeared to have made such a huge difference to referral practices - to the extent of the almost complete sidelining of a service in one site, which appeared an integral part of the service in another:

\section{'We have a very good rapport with the Macmillan nurse to the point where, if anything, we tend to accept her advice, we are probably the ones asking her more, you know, and going along with what she says because obviously I know, and I have worked with her for years.' (DN14)}

Responders seldom mentioned the impact of specialist nursing services on patients. What appeared more critical was the relationship that existed between the professionals - be it a difficult relationship based on poor communication and trust, or a facilitative relationship based on mutual respect and trust. It is known that general and specialist palliative care professionals can adopt a 'them and us' mentality, especially where there are communication breakdowns and over-stressed services, ${ }^{26}$ and palliative care nurses can talk in such a way that professionals who do not work in the same settings are seen as 'others'. ${ }^{33}$

GPs could also question their own personal need to refer to specialist services:

'I don't refer patients directly to the Macmillan nurses, don't know, don't know what they can offer that I can't' (GP5)

'I know that the hospice-at-home team have struggled in the past to almost make a role for themselves, I think sometimes the district nurses have said, well, what are they doing, they are just duplicating what we are doing' (SN6)

Criticisms of specialist services by GPs and district nurses were identified over 15 years ago, ${ }^{34,35}$ with concerns voiced about services eroding generalist roles and taking over care. It is notable that the findings of this study are similar, despite the many changes in primary care over that time.

\section{Game-playing}

Where the relationship with a fellow professional had deteriorated, responders appeared adept at 'getting round' them to achieve a particular aim. Professionals carefully presented issues to achieve a desired outcome, or bypassed 'difficult' professionals:

'As a district nurse my other way of getting past that is because I am like a Rottweiler, I don't let go ... I do find that if you have got a foot in the door at the hospice with a patient, if the situation with the GP unfortunately breaks down, there are other avenues to go down ... that sounds like we are all playing games, doesn't it really, but sometimes that is the way.' (SN6)

'Certainly then if we as independent prescribers could prescribe everything, we actually wouldn't then really need the GP because there is no problem about us referring to hospitals, no problems about us getting patients seen early, we actually don't need the GP if they are not willing to cooperate. There is a back door to get everything done if we need it done, apart from the initial prescribing at home.' (SN8)

'I don't think with this particular person [GP] it [referrals] will ever change, but you just have to find back door, ways of doing it, like 
encouraging, er, the practice nurse to get involved, and refer on if she finds anything out. Sounds very underhand, doesn't it, but sometimes needs must.' (DN11)

Similes were employed for using existing systems to achieve aims covertly - 'back door', 'playing games', 'getting what I want' - and all implied staff members had prior knowledge of the systems, priorities, and preferences of others with whom they worked.

Other research has shown that nurses may use strategies to 'cut' GPs out of assessment and prescription processes, but do work together when having to deal with a difficult patient. ${ }^{25}$ Acknowledgement was made that others involved in the care of that patient were aware of this gameplaying, and could collude with it.

\section{DISCUSSION}

\section{Summary of main findings}

This study describes how judgements about fellow professionals affected choices about collaborative working practices within community palliative care services. Judgements were based on previous experiences of working with individual professionals and, usually, on an assessment of their competence in such areas as communication, respect they showed towards others, working practices, perceived expertise, and perceptions of elitism. Negative perceptions of a fellow professional frequently resulted in 'game-playing' to allow working in a way that ameliorated the effect of that professional's working practices or bypassed them entirely.

\section{Comparison with existing literature}

This study is important because it highlights the importance of a previously under-explored aspect of collaborative working within community palliative care services. Few studies of referrals have explored the influence of professional factors, such as judgements; most choose to focus on patient factors, such as demographic characteristics. ${ }^{7,8}$

Studies of teamwork and collaborative working have identified that team co-location (teams based together in the same building or area), size and composition, organisational support, clear objectives, mutual trust, and professional cultures are factors that facilitate or impede collaboration..$^{31,36}$ Many of these issues, such as co-location and access, are supported by this data but, importantly, the impact of positive and negative judgments about fellow professionals on collaborative working does not appear to be an issue previously explored in depth.
It is unsurprising that responders had a tendency to make negative judgements about the actions of others. There are clear indications, particularly in psychological literature, that people are more likely to attribute positive events to themselves and dismiss negative events as attributable to other causes or people - the 'self-serving attributional bias'. ${ }^{37}$ People tailor their judgements of others to affirm their own self-worth and that of their group, and ensure their evaluations of others place themselves and their own attributes in a positive light. . $^{38,39}$

In addition to these self- and group-serving behaviours, there is strong evidence that people perceive their own actions in a different way to those of others. People consider their own behaviour to be influenced by the situation and the pressures exerted by circumstances, whereas they are more likely to believe the actions of others are influenced by stable traits such as character or temperament. ${ }^{40}$ These theoretical perspectives appear to be supported by our findings. For example, those not in a persons own professional grouping are referred to above as lacking in expertise, elitist, or uncooperative - all more stable trait dispositions rather than being influenced by a particular situation. These insights may help to understand professionals' behaviours and judgements, but do not negate the impact that these actions have on collaborative working.

Collaborative working appears to be influenced by professionals' interpersonal and interprofessional relationships. That there are interprofessional differences is not surprising as practitioners from different disciplines, with different histories, experiences and interests, are likely to understand practice problems and situations in different ways.

The relationship between doctors and nurses has been extensively examined ${ }^{41}$ and, certainly, the complexity of power relationships between doctors and nurses is highlighted in the present study. Nurses reported subtle interactions with doctors to maintain working relationships while achieving desired aims, subverting medical authority by bypassing normal procedures and, yet, appearing subservient in their observed interactions with doctors.

\section{Strengths and limitations of the study}

The strengths of this study include flexible data collection from multiple sources. Seeking multiple perspectives from different professionals about the same phenomenon more readily revealed complexities of judgements and referral practices. The study also gains from being a cross-case analysis of practices considered first in context. Although individual cases were very different, there were similar issues underlying referral decision making, enhancing the utility of findings to others. 
The study is partly limited by its size and the difficulties recruiting some participants, particularly GPs. Those GPs who participated did so only after more invitations to participate were sent and direct contact was made. Most cited workload as a barrier to participation. It is not possible to know whether the opinions of those finally recruited differed from those who did not participate.

Other limitations include the focus on healthcare rather than social care services; a focus on referrals within community settings, such that the views of those working in other settings are not considered; and the limited opportunities to collect observational data.

\section{Implications for clinical practice and future research}

This study suggests that further investigation of professional influences on collaborative working within community palliative care services is important. Primary care teams have continued to change in structure and function, with new policy and contractual obligations, and the redistribution of work between professionals. ${ }^{42,43}$ Initiatives such as the Gold Standards Framework ${ }^{44}$ continue to focus attention on community palliative care and the importance of collaboration. Understanding how such positive and negative judgements of fellow professionals potentially impacts on collaborative working in such a changing environment is important if such changes are to be effectively implemented.

Future palliative care policies and protocols need to take account of these complex and subtle influences on referrals and collaborative working. Increasingly collaboration, partnership, and teamwork are mandated within such policies ${ }^{45}$ and, yet, critical judgements about fellow professionals indicate that such partnerships may be difficult or fragile. Partnership may be helpful only when there is a clear rationale or explicit benefit with minimal costs. Actions to promote teamwork may promote referrals, so sharing working space or bases, sharing documentation, and spending social time together may have an impact on referral practices.

This study also has wider implications: primary care professionals frequently care for those with complex health problems in similar networks to those that palliative care professionals use. It may be that similar issues of judgement affect the way that primary care professionals work in other areas. In addition, the implementation of new roles, such as community matrons, may exacerbate or create collaborative problems. It is likely that similar judgemental attitudes equally affect such collaborations and may be important to consider in other fields.

\section{Funding body}

Catherine Walshe was funded by a Primary Care Researcher Development Award from the National Institute of Health Research, Department of Health (RDA02/020)

\section{Ethical approval}

Research ethics approval for the study was granted by Bolton Research Ethics Committee (03/BN/738)

\section{Competing interests}

The authors have stated that there are none

\section{Acknowledgements}

With thanks to all those who facilitated and participated in the research.

\section{Discuss this article}

Contribute and read comments about this article on the Discussion Forum: http://www.rcgp.org.uk/bjgp-discuss

\section{REFERENCES}

1. Sepúlveda C, Marlin A, Yoshida T, Ullrich A. Palliative care: the World Health Organization's global perspective. J Pain Symptom Manage 2002; 24(2): 91-96.

2. Department of Health. Our health, our care, our say. London: The Stationery Office, 2006.

3. NICE. Improving supportive and palliative care for adults with cancer: the manual. London: NICE, 2004

4. Farrell MP, Schmitt MH, Heinemann GD. Informal roles and the stages of interdisciplinary team development. J Interprof Care 2001; 15(3): 281-295.

5. Ouwens M, Wollersheim H, Hermens R, et al. Integrated care programmes for chronically ill patients: a review of systematic reviews. Int J Qual Health Care 2005; 17(2): 141-146.

6. Mitchell G, Del Mar C, Francis D. Does primary medical practitioner involvement with a specialist team improve patient outcomes? A systematic review. Br J Gen Pract 2002; 52(484): 934-939.

7. Ahmed N, Bestall JC, Ahmedzai SH, et al. Systematic review of the problems and issues of accessing specialist palliative care by patients, carers and health and social care professionals. Palliat Med 2004; 18(6): $525-542$

8. Grande GE, Addington-Hall JM, Todd CJ. Place of death and access to home care services: are certain patient groups at a disadvantage? Soc Sci Med 1998; 47(5): 565-579.

9. Catt S, Blanchard M, Addington-Hall J, et al. Older adults' attitudes to death, palliative treatment and hospice care. Palliat Med 2005; 19(5): 402-410.

10. Koffman J, Burke G, Dias A, et al. Demographic factors and awareness of palliative care and related services. Palliat Med 2007; 21(2): 145-153.

11. Gulzar l. Access to health care. Image J Nurs Sch 1999; 31(1): 13-19.

12. Aitken A. District nurses' triggers for referral of patients to the Macmillan nurse. Br J Community Nurs 2006; 11(3): 100-107.

13. Bestall JC, Ahmed N, Ahmedzai SH, et al. Access and referral to specialist palliative care: patients' and professionals' experiences. Int $\mathrm{J}$ Palliat Nurs 2004; 10(8): 381-389.

14. McNeilly DP, Hillary K. The hospice decision: psychosocial facilitators and barriers. Omega Journal of Death and Dying 1997; 35(2): 193-217.

15. Becker JE. Oncology social workers' attitudes toward hospice care and referral behavior. Health Soc Work 2004; 29(1): 36-45.

16. Weggel JM. Barriers to the physician decision to offer hospice as an option for terminal care. WMJ 1999; 98(3): 49-53.

17. Hanson LC, Sengupta S, Slubicki M. Access to nursing home hospice: perspectives of nursing home and hospice administrators. J Palliat Med 2005; 8(6): 1207-1213.

18. Fellowes D, Goodman M, Wilkinson S, et al. District nurses' referrals to home-based palliative nursing services. Nurs Times 2003; 99(14): $34-37$.

19. Todd CJ, Grande GE, Barclay SI, Farquhar MC. General practitioners' and district nurses' views of hospital at home for palliative care. Palliat Med 2002; 16(3): 251-254.

20. Walshe CE, Caress AL, Chew-Graham C, Todd CJ. Case studies: a research strategy appropriate for palliative care? Palliat Med 2004; 18(8): 677-684.

21. Yin RK. Case study research: design and method. 3rd edn. Thousand Oaks: Sage, 2003.

22. Goddard M, Smith P. Equity of access to health care services: theory and evidence from the UK. Soc Sci Med 2001; 53(9): 1149-1162. 
23. Ritchie J, Spencer L. Qualitative data analysis for applied policy research. In: Bryman A, Burgess RG, (eds). Analysing qualitative sata. London: Routledge, 1994.

24. Ingleton C, Hughes $P$, Noble B, et al. An evaluation of a Macmillan GP clinical facilitator project: the post-holders' perspective. Primary Health Care Research and Development 2003; 4(2): 177-186.

25. Street A, Blackford J. Communication issues for the interdisciplinary community palliative care team. J Clin Nurs 2001; 10(5): 643-650.

26. Burt J, Shipman C, Addington-Hall J, White P. Palliative care: perspectives on caring for dying people in London. London: King's Fund, 2005.

27. May C. Nursing work, nurses' knowledge, and the subjectification of the patient. Sociol Health Ill 1992; 14(4): 472-487.

28. Newton J, Waters V. Community palliative care clinical nurse specialists' descriptions of stress in their work. Int J Palliat Nurs 2001; 7(11): $531-540$.

29. Goodman C. The use of metaphor in district nursing: maintaining a balance. J Adv Nurs 2001; 33(1): 106-112.

30. Arber A. 'Pain talk' in hospice and palliative care team meetings: an ethnography. Int J Nurs Stud 2006; 44(6): 916-926.

31. Hall P. Interprofessional teamwork: professional cultures as barriers. $J$ Interprof Care 2005; 19(Suppl 1): 188-196.

32. Speed S, Luker K. Getting a visit: how district nurses and genera practitioners 'organise' each other in primary care. Sociol Health Illn 2006; 28(7): 883-902.

33. Li S. Doing criticism in 'symbiotic niceness': a study of palliative care nurses' talk. Soc Sci Med 2005; 60(9): 1949-1959.

34. Cartwright A. The relationship between general practitioners, hospita consultants and community nurses when caring for people in the last year of their lives. Fam Pract 1991; 8(4): 350-355.
35. Haste FH, Macdonald LD. The role of the specialist in community nursing: perceptions of specialist and district nurses. Int J Nurs Stud 1992; 29(1): 37-47.

36. Xyrichis A, Lowton $\mathrm{K}$. What fosters or prevents interprofessional teamworking in primary and community care? A literature review. Int J Nurs Stud 2007; Epub: doi:10.1016/j.ijnurstu.2007.01.015

37. Mezulis AH, Abramson LY, Hyde JS, Hankin BL. Is there a universal positivity bias in attributions? A meta-analytic review of individual, developmental, and cultural differences in the self-serving attributional bias. Psychol Bull 2004; 130(5): 711-747.

38. Sherman DK, Kim HS. Is there an 'I' in 'team'? The role of the self in group-serving judgments. J Pers Soc Psychol 2005; 88(1): 108-120.

39. Beauregard KS, Dunning D. Turning up the contrast: self-enhancement motives prompt egocentric contrast effects in social judgments. J Pers Soc Psychol 1998; 74(3): 606-621.

40. Watson D. The actor and the observer: How are their perceptions of causality divergent. Psychol Bull 1982; 92(3): 682-700.

41. Svensson R. The interplay between doctors and nurses - a negotiated order perspective. Sociol Health Illn 1996; 18(3): 379-398.

42. Charles-Jones H, Latimer J, May C. Transforming general practice: the redistribution of medical work in primary care. Sociol Health Illn 2003 25(1): 71-92.

43. Jones L, Green J. Shifting discourses of professionalism: a case study of general practitioners in the United Kingdom. Sociol Health Illn 2006; 28(7): 927-950

44. Thomas K. The Gold Standards Framework in Community Palliative Care. Eur J Palliat Care 2003; 10: 113-115.

45. Walshe C, Caress A, Chew-Graham C, Todd C. Evaluating partnership working: lessons for palliative care. Eur J Cancer Care (Engl) 2007; 16(1): 48-54. 